\title{
A Novel Method Providing Multimedia Contents According to Preference Clones in Mobile Environment*
}

\author{
Sanggil Kang ${ }^{1}$ and Sungjoon Park ${ }^{2, * *}$ \\ ${ }^{1}$ Computer Science and Engineering, INHA University, 253 Younhyun-dong, Nam-gu, \\ Incheon, South Korea \\ sgkang@inha.ac.kr \\ ${ }^{2}$ Department of Mobile Game, Kongju Communication and Arts College, Gongju, Chungnam \\ 314-713, South Korea \\ sjpark@kcac.ac.kr
}

\begin{abstract}
We introduce a novel method of automatic providing multimedia content in mobile environment. With using our method, the problem of the limitation of resource of mobile devices can be solved. In this paper, we introduce a novel method of recommendation of personalized contents according to preference clones using a collaborative filtering technique in mobile environment. We divide the user group to two sub-groups by analyzing the match of preferences of members in the sub-groups. The division process recursively applies to each sub-group and place the sub-groups in a binary decision tree (BDT). From the binary decision tree, we identify the preference clones of each target user by matching the target user's consumption behavior to that of each sub-group in the BDT with a sequential manner. We also implemented our system based on Java Micro Edition platform.
\end{abstract}

Keywords: Binary decision tree, mobile devices, personalization, and preference clones.

\section{Introduction}

By the rapid spread of mobile devices such as cellular phone, PDA, and portable TV, user can enjoy many kinds of multimedia contents through mobile appliances anytime and anywhere. Especially, the population of Digital Multimedia Broadcasting (DMB) user increases drastically year by year since available as a commercial service from May 2005 in Korea. Also, DMB service has been at the center of developing issues in mobile environment. Because of significant increase of contents through mobile devices, user sometimes spends heavy effort and time for searching and downloading their preferred contents.

In order to solve the problem, we introduce a novel method of recommendation of personalized contents according to preference clones using a collaborative filtering

\footnotetext{
* This work was supported by INHA UNIVERSITY Research Grant (INHA-35035-01).

** Correspondence author.
} 
technique in mobile environment. In this paper the preference clone means a user whose consumption pattern is very similar to that of another user. We divide the user group to two sub-groups by analyzing the match of preferences of members in the sub-groups. The division process recursively applies to each sub-group and place the sub-groups in a binary decision tree (BDT). From the binary decision tree, we identify the preference clones of each target user by matching the target user's consumption behavior to that of each sub-group in the BDT with a sequential manner.

The remainder of this paper is organized as follows. Section 2 introduces related work. Section 3 overviews the overall architecture of the proposed system. In Section 4, we explain our proposed method. In Section 5, we design and implement the prototype of the proposed system and show the performance comparison between our method and a conventional method. We then conclude our paper in Section 6.

\section{Related Work}

Various personalization techniques can be classified into three possible categories such as the rule-based, inference method, and collaborative filtering. The rule-based recommendation is usually implemented by a predetermined rule, for instance, if then rule. Kim et al. [1] proposed a marketing rule extraction technique for personalized recommendation on internet storefronts using tree induction method. As one of representative rule-based techniques, Aggrawall et al. [2] proposed a method to identify frequent item sets from the estimated frequency distribution using association-rule mining algorithm. The inference method is the technique that a user's content consumption behavior is predicted based on the history of personal content consumption behaviors. Lee [3] designed an interface agent to predict a user's resource usage in the UNIX domain by the probabilistic estimation of behavioral patterns from the user behavior history. Collaborative filtering (CF) technique recommends a target user the preferred content of the group whose content consumption mind is similar to that of the user. Because of the mature of the technique, $\mathrm{CF}$ has been attractive for predicting various preference problems such as net-news [4], e-commerce [5], digital TV [6], digital libraries [7], etc.

Several papers applied CF to mobile devices. Coster et al. [8] used a CF for predicting rating changes of movie data based on users' new or updated ratings in their system, MobiTip [9]. Also, Miller et al. [10] developed a recommender system for recommending video movie to occasionally connecting users through mobile devices. Tveit [11] applied a CF to mobile commerce based on using votes or opinions about products and services from similar customers using Peer-to-Peer. Zhang et al. [12] explored effective ways of delivering personalized content under the restrictions on wireless networks and mobile devices.

The literatures mentioned above do not consider recent implicit usage behavior for inferring users' preference. However, it can be a critical clue for predicting a target user's usage behavior near future. In this paper we use target user's implicit feedback by using binary decision technique. From the following section, we demonstrate our method in detail. 


\section{Architecture of Our System}

Fig. 1 shows the overall architecture of our system in mobile environment. The architecture is composed of two parts: one is server side and the other is client side.

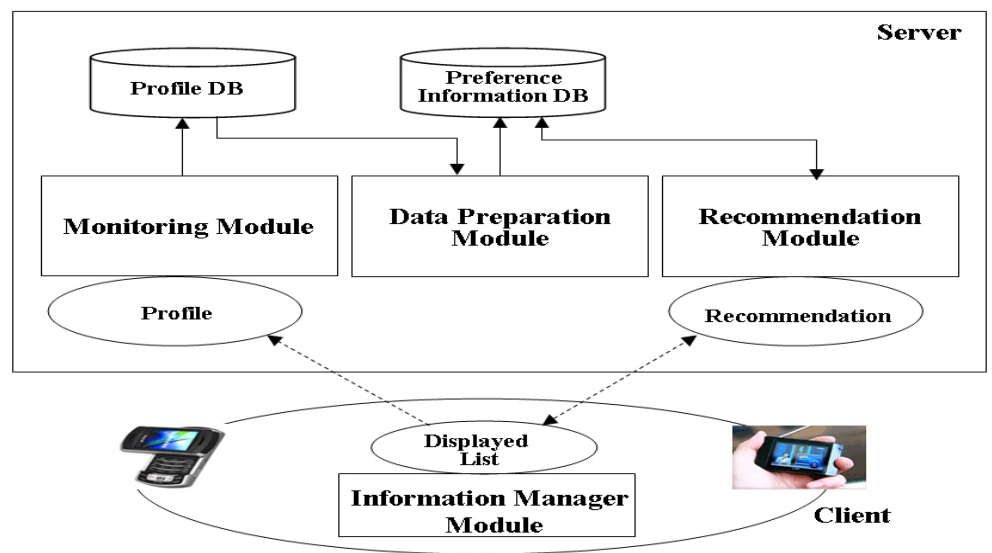

Fig. 1. Overall architecture of our system

The server side consists of three parts such as Monitoring Module (MM), Data Preparation Module (DPM), and Recommendation Module (MM). The MM records the $\log$ file such as user ID, gender, age, content consumption behavior (usage history), and store them into the Profile DB. The DPM eliminates nosy data from the collected usage history and makes groups according to users' age and gender. Also, it stores them into the Preference Information DB. The RM analyzes the preference of multimedia contents according to users' age and gender group using the proposed algorithm explained in detail from the following section. Then, it recommends preferred contents to each target user.

The client side has one module called Information Manager Module (IMM). The IMM displays recommendation list provided from the server side. Also, it records information of content consumed by the mobile user.

\section{The Proposed Recommendation Algorithm}

\subsection{Group and Member Preference}

In general the similarity of user preference can be characterized by age and gender. For instance, the documentary programs of DMB are usually popular favor for thirties and forties man, while the show programs are for teenagers and twenties. In each group, for each user, very similar or identical users to the user can exist, in the sense of consumption pattern. We call those similar or identical users "preference clones" in this paper. If preference clones for a new mobile user are founded, we recommend or provide the preferred contents of the preference clones to the new user, sometimes 
called "target user." To recommend or provide preferred contents to target user, we first group existing users by age and gender as seen in (1).

$$
U=\left[U_{1}, U_{2}, \cdots, U_{i}, \cdots U_{L}\right]
$$

where, $U_{i}(i=1,2, \ldots L)$ is the $i^{\text {th }}$ age and gender group in the whole existing reference group, $U$. Also, the $U_{i}$ includes $J$ members as expressed in (2).

$$
U_{i}=\left[u_{1}, u_{2}, \cdots, u_{j}, \cdots u_{J}\right]
$$

where, $u_{j}$ is the $j^{\text {th }}$ member in sub-reference group $U_{i}$. For $U_{i}$, the preference of contents can be computed and ordered by the frequency of the group's content consumption during predetermined time period. The overall preference order of contents for $J$ members can be denoted as $\operatorname{Pref}_{U i}$. Also, for member $u_{j}$, the preference of contents can be computed and ordered from high preference to low preference by the frequency of the member's content consumption. The preference order of the contents can be denoted as Pref $_{u j}$. Using the preference order of each group and its corresponding members, the preference clones can be found by making sub-groups in binary decision tree (BDT).

\subsection{Sub-reference Groups in Binary Decision Tree (BDT)}

From any sub-reference group $U_{i}$, we can also decompose it into two sub-groups by matching between the first element of $\operatorname{Pref}_{U i}$, denoted as $\operatorname{Pref} f_{U i}(1)$ and that of $\operatorname{Pref} f_{u j}$ of each member $u_{j}$, denoted as $\operatorname{Pref}_{u j}(1)$, as seen in (3).

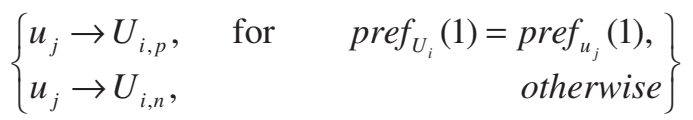

where, $U_{i, p}$ and $U_{i, n}$ is called positive sub-reference group and negative sub-reference group of $U_{i}$, respectively. Also, $j=1,2, \ldots ., J$. With the same process, $U_{i, p}$ can also decompose two sub-reference groups. Let's say, if there are $P$ members in the subreference group $U_{i, p}$ then the overall preference of contents consumed by the $P$ members can be computed and ordered. The ordered preferences of the sub-reference group can be denoted as $\operatorname{Pref} f_{U i, p}$. Also, for each member $k$ in the sub-reference group, his/her ordered preference for the contents can be denoted as $\operatorname{Pref} f_{u k}$. The $U_{i, p}$ can be decomposed by matching between the second element of $\operatorname{Pref}_{U i, p}$, denoted as $\operatorname{Pref}_{U i, p}(2)$ and that of $\operatorname{Pref}_{u k}$ of each member $u_{k}$, denoted as $\operatorname{Pref}_{u k}(2)$, as seen in (4).

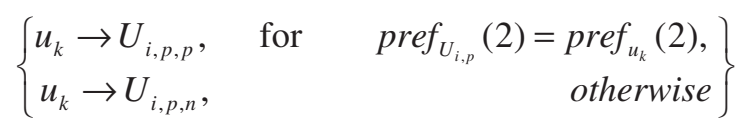

where, $U_{i, p, p}$ and $U_{i, p, n}$ is called positive sub-reference group and negative subreference group of $U_{i, p}$, respectively. Also, $k=1,2, \ldots, K$. For the same way, $U_{i, n}$ can also decomposed as seen in (5).

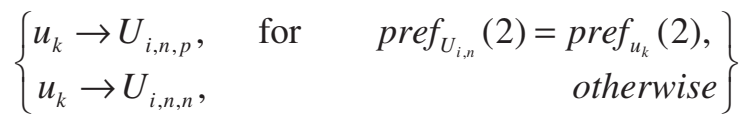


Where, $U_{i, n, p}$ and $U_{i, n, n}$ is called positive sub-reference group and negative subreference group of $U_{i, n}$, respectively. Also, $k=1,2, \ldots, J-K$. With the same process, each sub-reference group can be decomposed until each sub-reference group can not be decomposed anymore.

The accuracy of our method depends on the size of reference group. If the size is big then the accuracy will increase because the reference group has high possibility to contain more precise preference clones. In the following section, we compare the performance of our method with the typical Top-N recommendation method by varying the size of reference group.

\section{Experiments}

\subsection{Experimental Environment}

We implemented our system using CLDC/MIDP based on Java ME platform and Java SDK in the windowsXP environment. In order to test our method, J2ME Wireless Toolkit Emulator was used.

Due to difficulty in collecting users' contents in mobile environment until present time, we used 2000 TV viewers' watching history for executing our algorithm instead of the DMB programs. The data was provided by AC Nielsen Korea, one of the authorized market research companies, from January 1, 2003 to April 30, 2003. The watching history was collected by the Set-Top Box installed in the users' houses. The Set-Top Box also recorded viewers' profile information such as age and gender, and watched programs. In the data, there are around 100 programs. Thus, the multimedia content is considered the program in this experiment.

\subsection{Demonstration of Our Method}

Fig. 2 shows the demonstration of our method according to target user's watching behavior. We structured the binary decision tree (BDT) using 10 teenage boys' watching history collected during one month as explained in the previous chapter. When a target teenage user connected to the server, program P1 as seen in Fig. 3(a) is recommended through his mobile because P1 is the first ranked one in the teenage boy reference group as seen in the preference table. If the target user plays P1 by clicking the 'play' button on the mobile which implies positive feedback of our recommendation then we move to the left of the next level and recommend P5 at the next stage as seen in Fig. 3(b). The P5 is the second ranked program in the subreference group whose all members watched P1. If the target user skips the recommend program by clicking the 'skip' button which implies negative feedback, we move to the right of the next level and recommend P2 as seen in Fig 3(c). The P2 is the second ranked program in the sub-reference group whose all members did not watch P5. If the target user plays P2 then we move to the left of the next level and can find the preference clone $u_{1}$ of the target user. The programs the preference clone watched are automatically recommended to the target user in the future. Like this, our method dynamically recommends programs to the target user in a recursive manner. 


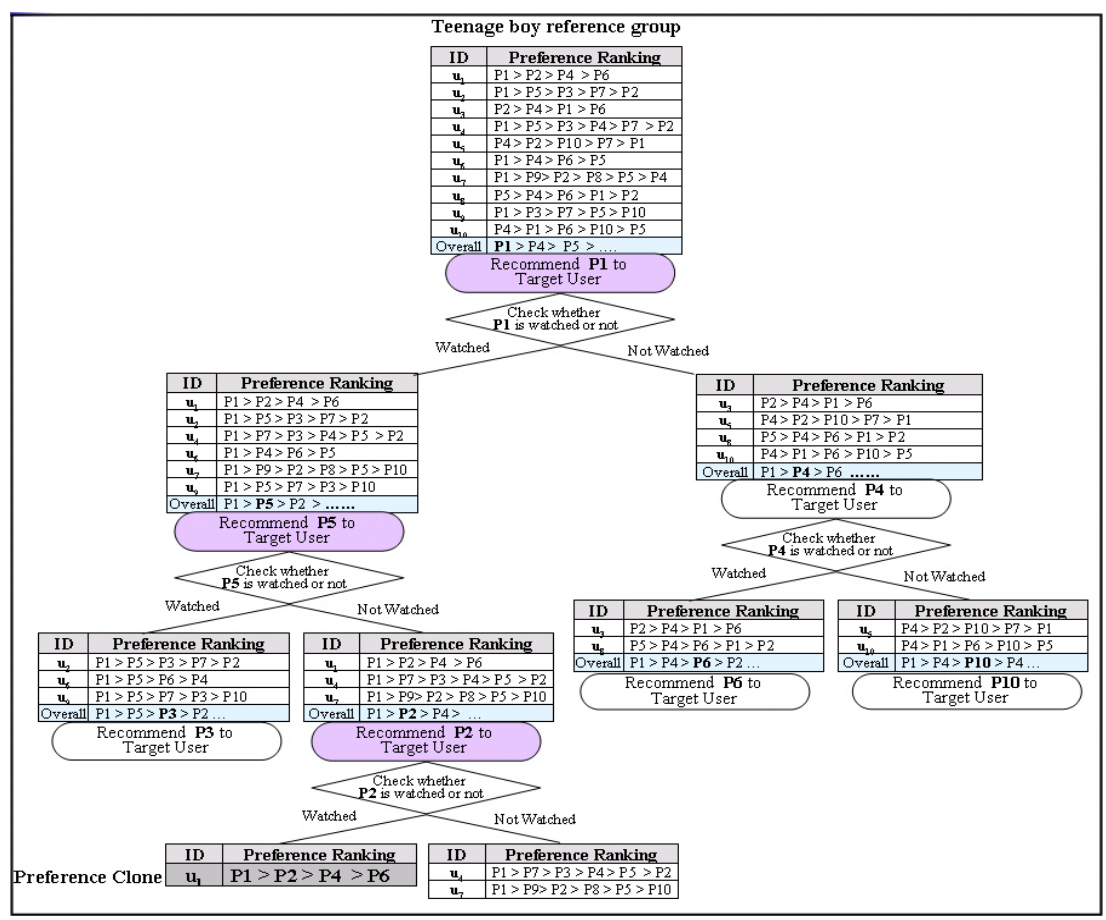

Fig. 2. The BDT of the teenage boy group

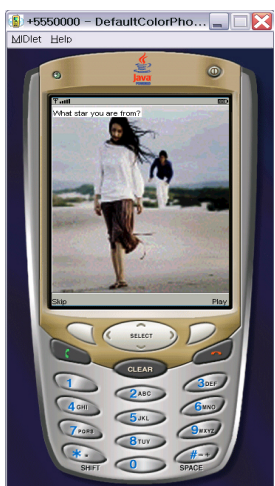

(a) The beginning stage

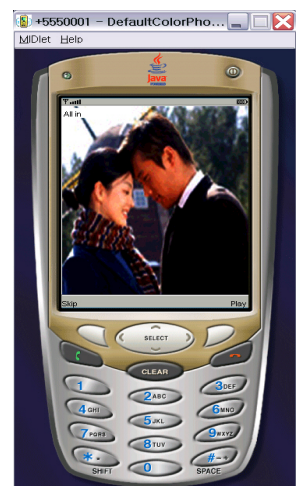

(b) The positive feedback stage

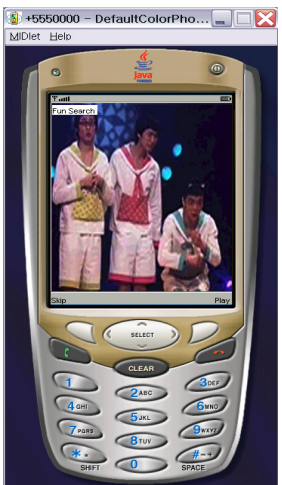

(c) The negative feedback stage

Fig. 3. The examples of dynamical program providing through the target user's mobile device

\subsection{Evaluation of Our Method}

In this section, we compared the performance of our method with that of the conventional recommendation method, TOP-N [13]. The 2000 TV viewers' watching history was divided into reference data and target user data. Also, the reference data was grouped according to its members' age and gender such as teenage boys, teenage 
girls, twenties man, twenties woman, thirties man, thirties woman, forties man, and forties woman. The performance was evaluated with the overall ratio of the number of match between recommended programs to target users' preference clones and watched programs by them. Also, we repeated the evaluation by varying the size of reference data such as 500,1,000, and 1,500 members in the reference data and the collection period of usage history such as one month, two months, and three months. For the performance evaluation, the one next month data of the reference data was used.

From the table, it is shown that our method outperforms TOP-N method when the reference data size is relatively big. However, when the reference size is relatively small, TOP-N is little better than our method. For instance, for 500 reference members with one month collection period, the accuracy of our method and TOP-N are $65 \%$ and $68 \%$, respectively. However, for 1,000 reference members, we have $73 \%$ for our method and $72 \%$ for TOP-N. For 1,500 reference members, we have $81 \%$ for our method and $73 \%$ for TOP-N. It is because the possibility that the preference clones of each target user are included in the reference data is low.

From the table, it is shown that the collection period does not much influence on the accuracy for both methods. For 1,500 reference data, the accuracies of our method for one month, two months, and three months are $81 \%, 82 \%$, and $84 \%$, respectively. Also, for TOP-N, we have $73 \%, 74 \%$, and $75 \%$. In general, user's preference trend for the multimedia content like TV program does not drastically change. That is the reason why the collection period does not give an impact on improving the performance for both our method and TOP-N method.

Table 1. Comparion of performances of our method and TOP-N method

\begin{tabular}{|c|c|c|c|c|}
\hline \multirow{2}{*}{$\begin{array}{c}\text { Data } \\
\begin{array}{c}\text { collection } \\
\text { period }\end{array}\end{array}$} & Method & 500 & 1,000 & 1,500 \\
\cline { 3 - 5 } 1 month & TOP-N & $68 \%$ & $72 \%$ & $73 \%$ \\
\cline { 2 - 5 } & Our & $\mathbf{6 5 \%}$ & $\mathbf{7 3 \%}$ & $\mathbf{8 1 \%}$ \\
\hline \hline \multirow{2}{*}{2 months } & TOP-N & $69 \%$ & $74 \%$ & $74 \%$ \\
\cline { 2 - 5 } & Our & $\mathbf{6 6 \%}$ & $\mathbf{7 4 \%}$ & $\mathbf{8 2 \%}$ \\
\hline \hline \multirow{2}{*}{3 months } & TOP-N & $69 \%$ & $75 \%$ & $75 \%$ \\
\cline { 2 - 5 } & Our & $\mathbf{6 6 \%}$ & $\mathbf{7 6 \%}$ & $\mathbf{8 4 \%}$ \\
\hline
\end{tabular}

\section{Conclusion}

In this paper, we introduced a novel method of providing automatic multimedia contents through mobile devices. From the result of the performance comparison between our method and the conventional method, we can conclude that the performance of our method is better than that of the conventional method for relatively large reference data. However, for small reference data, our method is not appropriate for use.

The data (2,000 viewers) we used for evaluating our method and the conventional method is not big enough to confirm the accuracies. Therefore, we need to redo the 
experiment with more data set. Also, we need to apply our method to other mobile multimedia contents such as music, mobile shopping mall, and mobile web service.

\section{References}

1. Kim, J.W., Lee, B.H., Shaw, M.J., Chang, H.L., Nelson, M.: Application of Decision-Tree Induction Techniques to Personalized Advertisements on Internet Storefronts. International Journal of Electronic Commerce, vol. 5, no. 3 (2001) 45-62

2. Aggrawall, R., Imielinski, T., Swami, A. : Mining Association Rules between Sets of Items in Large Databases. Proc. ACM SIGMOD Int'l Conference on Management of Data (1994) 207-216

3. Lee, J.J. : Case-based plan recognition in computing domains. Proc. The Fifth International Conference on User Modeling, (1996) 234-236

4. Resnick, P., Lacovou, N., Suchak, M., Bergstrom, P., Riedl, J. : GroupLens: An Open Architecture for Collaborative Filtering of Netnews. Internet Research Report, MIT Center for Coordination Science (1994) http://www-sloan.mit.edu/ccs/1994wp.html

5. Schafer, J.B., Konstan, J., Riedl, J. : Recommender systems in e-commerce. ACM Conference on Electronic Commerce (1999) 158-166

6. Cotter, P., Smyth, B. : Personalization Techniques for the Digital TV world. Proc. European Conference on Artificial Intelligence (2000) 701-705

7. Bollacker, K.D., Lawrence S., Giles, C.L.: A System for Automatic Personalized Tracking of Scientific Literature on the Web. Proc. ACM Conference on Digital Libraries (1999) $105-113$

8. Coster, R., Svensson M. : Incremental Collaborative Filtering for Mobile Devices. Preceedings of the 2005 ACM Symposium on Applied Computing (2005) 1102-1106

9. Rudstrom, S., Svensson, M., Coster, R., Hook, K.: MobiTip: Using Bluetooth as a Mediator of Social Context. $6^{\text {th }}$ International Conference of Ubiquitous Computing (2004)

10. Miller, B.N., Albert, I., Lam, S.K., Konstan, J.A., Riedl, J.: Movielens Unplugged: Experiences with an Occasionally Connected Recommender System. Proceedings of the $8^{\text {th }}$ International Conference on Intelligent User Interfaces (2003) 263-266

11. Tveit, A.: Pear-to-peer based Recommendations for Mobile Commerce. Proceedings of the $1^{\text {st }}$ International Workshop on Mobile Commerce (2001) 26-29

12. Zhang, D., Shijagurumayum, S.: Personalized Content Delivery to Mobile Devices. IEEE International Conference on Systems, Man and Cybernetics vol. 3 (2003) 2533-2538

13. Mukund D., George K.: Item-Based Top-N Recommendation Algorithms, ACM Transactions on Information System, vol. 22, Issue 1 (2004) 143-177 